\section{VARIATIONS DU NIVEAU MARIN \\ ET PHASES MORPHOCLIMATIQUES DANS LE DÉSERT DU NAMIB CENTRAL, AFRIQUE DU SUD-OUEST}

\section{INTRODUCTION}

De décembre 1971 à avril 1972, nous avons effectué des recherches ( ${ }^{1}$ ) concernant la morphogenèse quaternaire des littoraux et de leur hinterland en Afrique occidentale (SénégalMauritanic) et en Afrique du Sud-Ouest (Namib Central) (fig. 1). Dans ces régions, elle résulte de l'interaction de processus littoraux, fluviatiles et éoliens. Des travaux importants ont été entrepris en Afrique occidentale par des géomorphologues et géologues français (TRICART 1961, MICHEL 1967, 1968, ElouARD 1967). Ils ont éclairci les aspects fondamentaux de la stratigraphie et de la morphogenèse régionales. Certains problèmes demeurent cependant non résolus. En Afrique du Sud-Ouest il n'existe encore aucune étude d'ensemble des problèmes que nous avons abordés. La bibliographie n'offre quelques renseignements que sur les plages soulevées (JAeger 1965, Davies 1959, SPREITzer 1965) et sur les terrasses fluviatiles (JAeger 1965). SoARes dF CARvalHo (1961) a décrit la stratigraphie et la géomorphologie des hauts niveaux marins du littoral du désert de Moçâmedes (Angola méridional). Ces niveaux présentent des dépôts coquilliers et sont peut-être comparables à ceux que nous avons étudiés.

Les deux régions ont une position géographique analogue (latitude, climat, courants marins, orientation du littoral).

(1) Nous remercions la «Deutsche Forschungsgemeinschaft» qui a soutenu nos recherches. Cet article est un extrait modifié de l'article RUST et WIENEKE, 1973a. Nous souhaitons ainsi soumettre nos résultats à la discussion des géographes francophones. Nous remercions Mme S. Daveau d'avoir bien voulu corriger notre texte.
Les recherches que nous avons effectuées en Afrique occidentale et surtout l'étude critique des coupcs-types et de l'interprétation qui en avait été donnée, nous ont suggéré des hypothèses de travail pour nos travaux en Afrique du Sud-

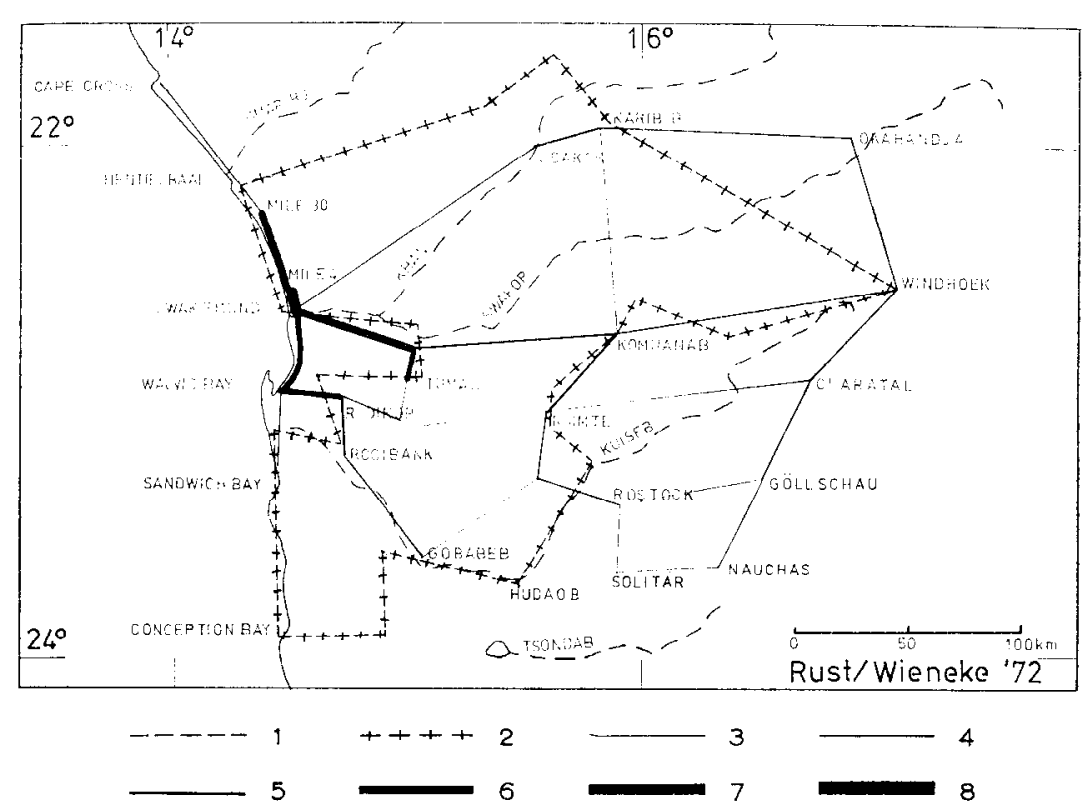

Fig. 1 - Itinéraires parcourus par les auteurs en Afrique du Sud-Ouest. 1: Rivières sèches, 2: survol, 3: itinéraire parcouru une fois, 4: 2 à

3 fois, 5: 4 à 7 fois, 6: 10 à 12 fois, $7: 13$ à 15 fois, 8: plus de 15 fois.

Ouest. Cependant, des différences importantes entre les deux régions étudiées (altitude de l'hinterland, extension des déserts, centres de glaciation différents dans les deux hémisphères), s'ajoutant à la grande distance qui les sépare, obligent à être très prudents dans les comparaisons.

La figure 2 de notre article (RUST et WIENEKE 1973a) montre les rapports existant entre les diverses recherches que nous avons effectuées dans le désert du Namib Central et les corrélations qu'on peut établir entre les résultats obtenus localement. On ne reprendra pas ici tous les problèmes abordés qui feront l'objet d'autres publications, mais seulement les résultats d'ensemble pour les soumettre à la discussion. 


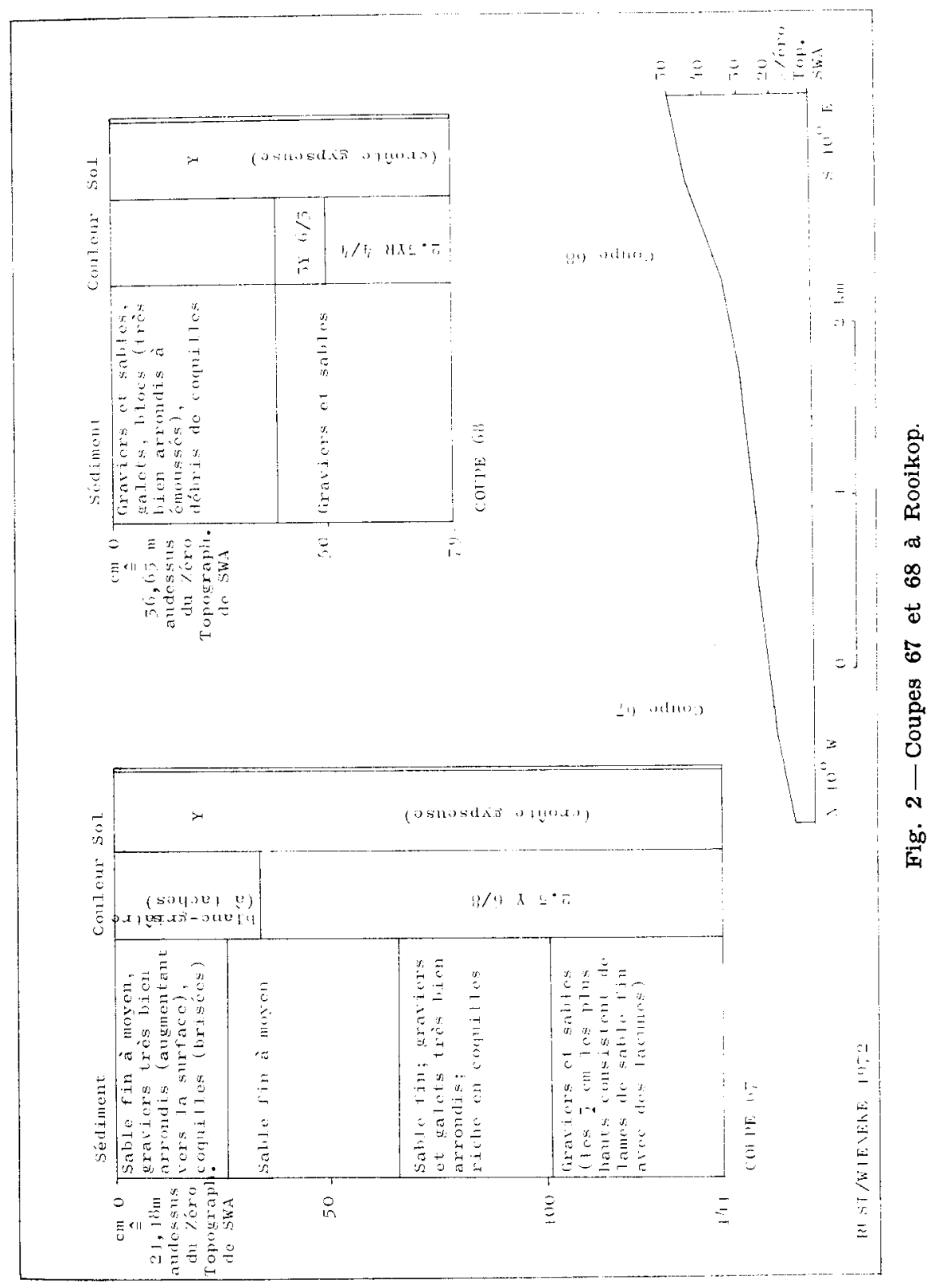

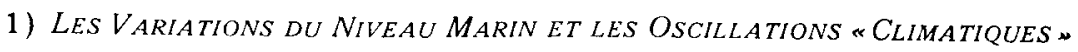

a) Les variations du niveau marin. - Nous admettons qu'entre deux niveaux marins subaériens étagés, le plus élevé est le plus ancien. Ce postulat d'une chronologie relative fondée sur la seule géomorphologie paraît valable pour la région étudiée, car on n'y connaît pas de mouvements tectoniques pour la période qui nous intéresse (Quaternaire récent) ${ }^{2}$ ).

En plusieurs endroits, nous avons observé deux niveaux marins indubitablement plus hauts que le niveau actuel. Nous les nommerons provisoirement niveau de $17 \mathrm{~m}$ (le plus ancien) et niveau de $2 \mathrm{~m}$ (le plus récent) ('). La coupe 67, à Rooikop (fig. 2), contient des sédiments témoignant d'une transgression et d'une régression du niveau marin de $17 \mathrm{~m}$ et, dans les deux cas, de la présence de la zone de déferlement à cet endroit (degré d'arrondissement et dimension des sédiments). Le dépôt de transgression contient surtout des coquilles entières, tandis que celui qui marque la régression n'offre que des coquilles brisées. Cela démontre vraisemblablement une modification des conditions écologiques. Les coquilles entières indiquent une avancée de la zone de déferlement qui a changé le milieu écologique (sable moyen et fin, de 26 à $66 \mathrm{~cm}$ de profondeur dans la coupe); puis la zone de déferlement traversa une deuxième fois ce même endroit au retrait de la mer. Cet ancien fond marin constitue actuellement une terrasse topographique.

Le niveau marin de $2 \mathrm{~m}$ est représenté par un récif de déferlement, depuis 12 miles au sud de Swakopmund jusqu'à Hentiesbaai. Le littoral correspondant a été reconnu à Mile 30 où il se trouve à $+2,5 \mathrm{~m}$ STL (WIENEKE et RUST, 1972). La figure 3 décrit ce niveau marin aux points de vue géo-

(2) Ce que nous écrivons sur la position des phases morphogénétiques dans la chronologie du Quaternaire reste encore provisoire. A l'aide de considérations morphologiques nous constatons que la séquence observée représente les évènements les plus récents. On peut probablement la ranger dans le Quaternaire récent. Peut-être les phases les plus anciennes remontent-elles au Quaternaire moyen.

( ${ }^{3}$ Ces indications d'altitude moyenne se réfèrent au niveau des marées de tempête, storm tide level (STL), à Mile 30, lequel est situé à $+3,43 \mathrm{~m}$ par rapport au zéro topographique du Sud-Ouest africain. 
morphologique (récif de déferlement, falaise), sédimentologique et paléontologique.

A Vineta, on peut prouver l'existence d'un bas niveau marin subactuel. A marée basse, un grès de plage (beach rock) affleure de la cote $-3,38$ à la cote $-2,94 \mathrm{~m}$ STL. Ce beach rock n'est pas inclus dans le récif de déferlement que la mer attaque aussi à cet endroit et est donc plus récent. Le fait qu'on trouve des galets de beach rock parmi les galets de la plage actuelle montre qu'il est cependant inactuel. RUSSEL (1962) a démontré que la cimentation du beach rock ne peut se faire qu'à l'air libre, au dessus du niveau de la marée la plus basse. Le niveau marin correspondant était donc inférieur à l'actuel.

Nous n'avons trouvé aucun autre niveau marin, subaérien ou submergé, en dehors de ceux qui viennent d'être décrits. La coupe 68, à Rooikop, (fig. 2) montre un sédiment marin reposant sur des graviers fluviatiles. L'altitude absolue de la coupe $(+33,22 \mathrm{~m}$ STL $)$ pose des problèmes d'interprétation. Le littoral correspondant est conservé sous forme d'une plage ancienne à galets, située à $40,47 \mathrm{~m}$ STL. L'application du postulat ci-dessus énoncé conduirait à admettre l'existence d'un niveau marin élevé, plus ancien que celui de $17 \mathrm{~m}$. Mais on ne peut exclure l'idée que la coupe 68 et la plage correspondent à l'extension maximale de la transgression responsable du dépôt de la formation marine de la coupe 67, observée à $+17 \mathrm{~m}$. En effet, une transgression est un phénomène qui se déroule progressivement dans le temps et, d'autre part, on n'observe entre les deux coupes aucun vestige d'une phase morphologique différente mais, au contraire, des coquilles affleurent en surface sur toute la pente entre les coupes 67 et 68 . On ne peut donc pas exclure qu'elles se rapportent à un même niveau marin.' Nous avons conscience qu'une telle interprétation va à l'encontre des idées généralement admises sur l'étagement des hauts niveaux marins. Il faut attendre, pour conclure, les résultats des datations au Carbone 14.

b) Les oscillations «climatiques». - Le tableau I contient les critères de terrain qui nous ont permis de déceler les phases «arides»ou «humides» dans l'évolution «climatique».
Les analyses de laboratoire encore non réalisées (granulométrie, morphoscopie ...) pourront peut-être fournir d'autres critères.

\section{TABLEAU}

Critères d'aridité et d'humidité observables sur le terrain

Critères d’《aridité»

processus

formation d'un reg (déflation)

ensablement éolien (par exemple dans les rigoles)

formes du relief

nebka

barkhanes

sédiments

sédiments de sebkha sables de barkhanes éolianite

sols croûtes gypseuses régosols (sur les nebka) «takyres»

Critères $d$ «humidité»

processus

formation de pentes dissection

formes du relief cônes alluviaux vallées

sédiments sédiments fluviatiles galets fluviatiles $\left(^{1}\right)$

(1) Cf. Rust et Wieneke, 1973b, où est discutée la possibilité d'utiliser les analyses d'arrondissement des galets pour en tirer des conclusions morphogénétiques «climatiques».

On peut tenter de mettre en corrélation la succession prouvée des phases «climatiques» pour la région côtière du désert du Namib Central et celle des phases eustatiques.

Le profil topographique du point Mile 30 (fig. 4) présente la succession de plusieurs phases «climatiques»: la mer de $17 \mathrm{~m}$ s'est avancée sur un dépôt de sable éolien cimenté («aridité»), puis les dépôts marins furent encroûtés par du gypse («aridité») après le retrait de l'océan. Une phase de dissection très intense a ensuite entraîné la formation de pentes («humidité»). La création d'un gradin dans les dépôts 
marins suppose une cimentation antérieure («aridité»); la pente formée lors de la phase «humide» fut encroûtée par du gypse ( «aridité») avant que la mer de $2 \mathrm{~m}$ envahisse une baie jusqu'à la limite marine de $+2,15 \mathrm{~m}$ STL.

Les oscillations climatiques postérieures peuvent être repérées au long d'un profil topographique au point Mile 4 (fig. 3). La mer de $2 \mathrm{~m}$ s'avança ici en entaillant une falaise dans les sédiments fluviatiles déposés lors de la phase «humide»

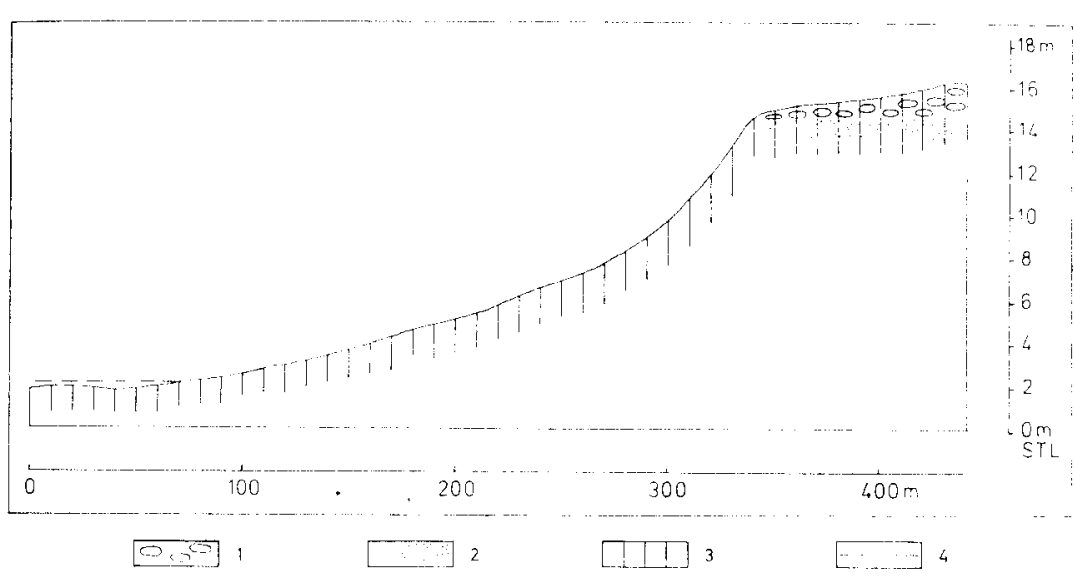

Fig. 4-Profil topographique du point Mile 30. 1: Galets marins,

2: éolianite (sable dunaire cimenté), 3: gypse, 4: niveau marin de $2 \mathrm{~m}$

précédente, ce qui implique un encroûtement gypseux antérieur («aridité»). La mer en régression déposa d'abord des sédiments marins, entre le récif de déferlement et le littoral. Ils furent recouverts par des sédiments de sebkha («aridité»), puis par des graviers fluviatiles ( $«$ humidité»), corrélatifs de la dissection de la falaise («humidité»). L'encroûtement gypseux de ces sédiments fluviatiles, la formation d'un reg de déflation et l'ensablement corrélatif des rigoles de la falaise, les nebka à régosols (Kupstendünen) témoignent de la phase actuelle («aridité»).

c) Corrélation des variations du niveau marin et des oscillations «climatiques». - MicheL (1967) a représenté les phases de morphogenèse «climatique» et de morphogenèse eustatique de la région du Sénégal inférieur par des courbes 


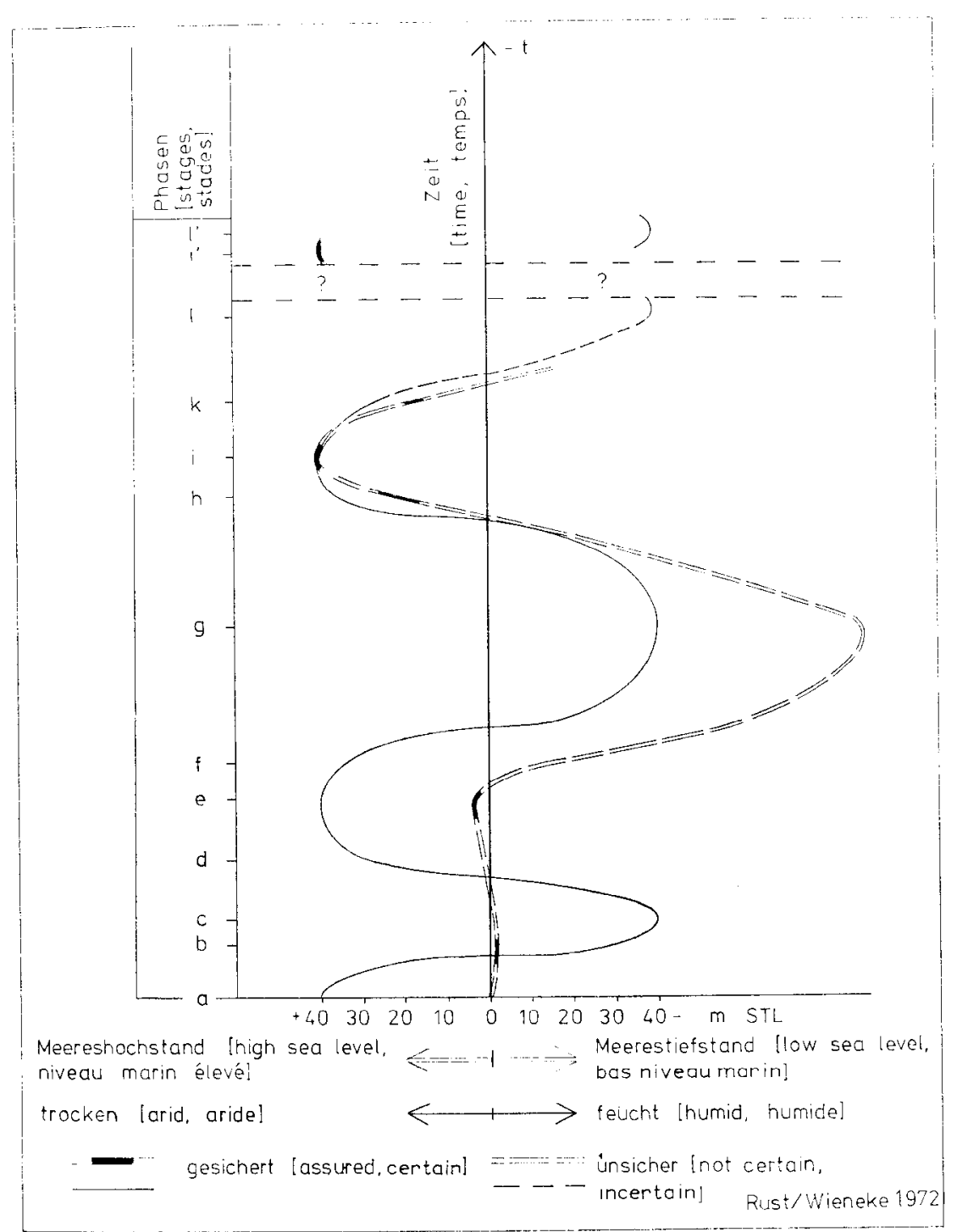

Fig. 5--Variations du niveau marin et oscillations climatiques dans le désert du Namib central. axées sur un vecteur représentant le temps. Suivant la méthode de MICHEL, nous avons construit les courbes de variation du niveau marin et des oscillations «climatiques» pour la même période du Quaternaire (fig. 5: a-l et l'). Provisoirement nous indiquons le temps sans échelle. Nous avons distingué des périodes certaines et d'autres hypothétiques. La courbe des variations du niveau marin construite sur une abscisse métrique n'est certaine que pour les périodes qui peuvent être repérées sans erreur en des points précis; on peut lire ainsi leur amplitude. Les intervalles entre ces troncons de courbe ne sont pas certains; on ne peut qu'indiquer la tendance transgressive ou régressive de ces variations.

Les courbes du diagramme sont fondées sur la succession des phases eustatiques et «climatiques». Ce diagramme de Michel que nous avons été les premiers à construire pour la région côtière du déscrt du Namib Central montre une corrélation des phases «arides» avec les derniers niveaux marins élevés (phases $\mathrm{k}$ à $\mathrm{h}$, e et a) et des phases «humides» avec les derniers bas niveaux marins ( $\mathrm{g}$ et $\mathrm{b}$ )

Il est peu probable que ce parallélisme soit un hasard; il indique au contraire une interaction de l'évolution glacioeustatique mondiale et de l'évolution «climatique» régionale.

Si l'on compare au diagramme construit pour le désert du Namib Central celui qu'a établi Michel (1967), on s'aperçoit que le parallélisme entre phases «arides» et niveaux marins élevés ne s'applique pas à la région Sénégal-Mauritanie, en dépit des positions géographiquement comparables des deux régions.

2) Les Phases d'une Morphogenèse «Climatique». Proposition d’une CONCEPTION AMÉLTORÉE.

a) Introduction aux problèmes. - La courbe de l'évolution «climatique» dans le diagramme de Michel résulte de l'organisation en une série temporelle des critères groupés dans le tableau I en fonction de leur nature (formes du relief, processus, sédiments, sols). Ce faisant, on établit une relation directe entre les critères observables sur le terrain et deux expressions climatologiques complexes, «aridité» et «humidité» (qui n'expriment pourtant que certains aspects du climat 
réel). Ceci, dans le but de tenter de reconstituer l'évolution climatique. Ce type de déduction n'est ni logique, ni exact.

Ce problème montre l'illogisme foncier de la géomorphologie climatique et même de toute tentative pour établir des corrélations entre formes du relief et types de climat. Beaucoup de publications concernant la géomorphologie climatique, surtout celle des régions arides, fondent leur raisonnement sur un rapport direct entre formes du relief et climat alors que celui-ci n'est qu'un facteur entre autres du milieu géomorphologique. On peut faire le même reproche au diagramme de MicheL.

WEISCHET (1969) a critiqué l'application directe du terme climatologique «aride» aux problèmes géomorphologiques. Il suggère d'essayer d'établir une définition géomorphologique de la notion d'aridité appliquée aux formes de relief, tout en tenant compte, si possible, des résultats climatologiques. Cela concerne aussi, naturellement, les termes «humide», «roid» et «chaud».

Tricart et CaIlleux (1965) ont développé l'idée d'une dépendance de la morphogenèse non seulement à l'égard des «processus élémentaires» azonaux mais aussi des «processus complexes» agissant au sein d'un «système morphogénétique» zonal où se combinent divers facteurs écologiques: climat, végétation, sols. Ils distinguent des systèmes morphologiques à prédominance physique (par exemple dans les régions arides) et des systèmes à prédominance biologique et pédologique (par exemple dans les forêts tropicales). Ils négligent ainsi l'importance de la pédogenèse «aride» (croûtes gypseuses, croûtes calcaires) comme expression d'une activité morphologique réduite.

Rust (1970, p. 153-161 et 164) en étudiant le problème des inselbergs en Afrique du Sud-Ouest, 'a essayé de concevoir la morphogenèse fluviatile du Namib Central d'un point de vue morphoclimatique. Il a introduit les notions de «surface pluviale», «ruissellement de surface pluviale» et «allogénie du ruissellement de surface pluviale» $\left(^{4}\right)$ pour inter-

(4) «Regenfläche», «Regenflächen-Spülung», «Allochthonie der Regenflächen-Spülung». préter la morphogenèse «aride» en fonction des formes du relief et des processus morphogénétiques.

De son point de vue global de géomorphologue-pédologue, ROHDENBURG (1970) a montré l'importance dans la morphogenèse des facteurs écologiques dont le climat n'est qu'un élément. Il a élaboré un système basé sur l'alternance de «périodes d'activité morphodynamique», durant lesquelles les processus morphogénétiques sont actifs, et de «périodes de stabilité morphodynamique», pendant lesquelles les processus morphogénétiques font place à une pédogenèse active. Ces périodes sont déterminées par les conditions écologiques. Cette conception de Rohdensurg donne au climat sa juste place en tant que facteur morphogénétique.

b) Les phases «morphoclimatiques» en région aride. Après avoir distingué les phases morphogénétiques du désert du Namib Central et les avoir représentées sur un diagramme de Micher, nous avons étudié les terrasses du Swakop. Nous avons constaté un creusement actif du Swakop (ce qui indiquait une phase «humide» en fonction des processus morphogénétiques) lors de la phase e, typiquement «aride» selon les critères sédimentologiques et pédologiques.

Cette contradiction apparente nous a montré la manière illogique dont avait été construit le diagramme. En essayant de résoudre ce problème, nous sommes arrivés à combiner, élargir et approfondir les conceptions de RUST (1970) et celles de ROHDENBURG (1970). Nous avons élaboré une conception nouvelle décrite ci-dessous, dans le tableau II. Nous avons essayé d'appliquer cette terminologie nouvelle à notre terrain d'étude et nous l'avons trouvée acceptable puisqu'elle nous a permis d'éclaircir les relations existant entre la géomorphologie climatique et les morphogenèses fluviatile, éolienne et marine (eustatique).

Nous avons déduit et vérifié, sur le terrain, l'existence de trois phases morphoclimatiques responsables de la morphogènese quaternaire dans le désert du Namib: phases d'activité humide, de stabilité aride et d'activité aride. On peut aisément imaginer l'existence d'une phase de «stabilité humide» dont témoigne le paysage à inselberg de la marge intérieure du désert du Namib. Elle doit être responsable 


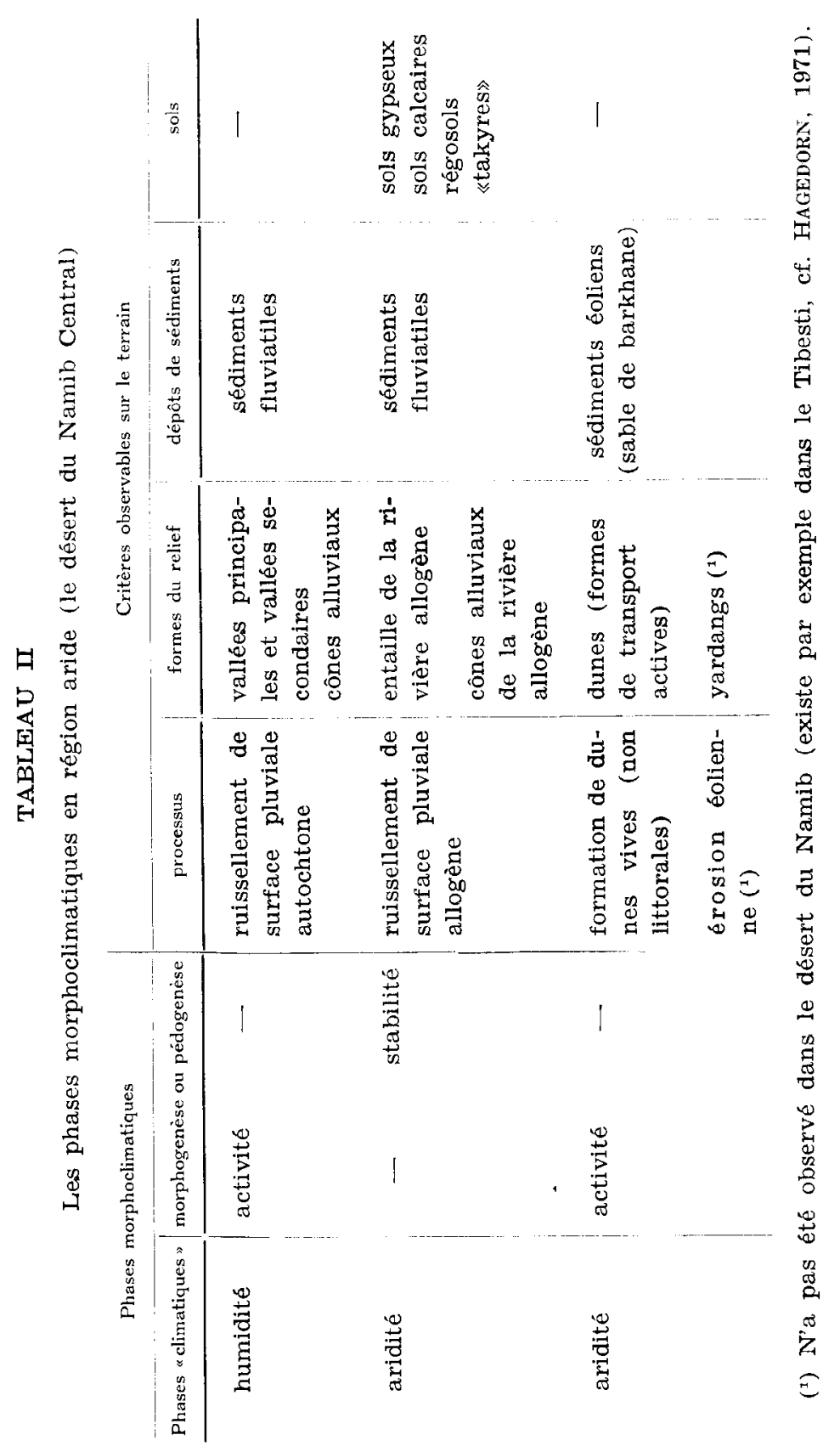

de la genèse de quelques autres formes du relief (ScHoLz, 1963, RUST, 1970). Mais nos recherches ne sont pas remontées jusqu'à l'époque des phases de «stabilité humide» (i).

L'analyse sédimentologique n'est pas décisive (tableau II). Les sédiments fluviatiles déposés lors des phases d'activité humide ou de stabilité aride sont analogues. Seule, l'analyse géomorphologique permet de distinguer ces deux phases: l'activité humide ne sera confirmée que si l'on prouve que les sédiments fluviatiles sont des dépôts corrélatifs d'une rivière autochtone.

On peut déduire du tableau II les divers types de morphogenèse provoqués par des successions différentes de phases morphoclimatiques. Ce problème sera repris dans WIENEKE et Rust (1973).

c) Application de cette conception nouvelle à la morphogenèse du désert du Namib Central. -- Cette nouvelle conception des phases morphoclimatiques en région aride permet d'expliquer la morphogenèse des terrasses du Swakop inférieur (RUST et WIENEKe, 1973a). Elles ont été formées lors d'une alternance de phases d'activité fluviatile allogène (rivières Swakop et Khan) et de phases d'activité fluviatile simultanément allogène et autochtone.

Afin de faciliter la comparaison entre les résultats obtenus en divers lieux, nous avons construit un diagramme de Michel modifié qui montre les phases de l'évolution littorale eustatique et les phases morphoclimatiques en région aride pour la région côtière du désert du Namib Central (fig. 6). Nous avons construit, du Sud au Nord, les courbes morphogénétiques de trois localités: Swakop inférieur, Mile 4 et Mile 30. Dans ce diagramme modifié, les notions d'«aridité» et $d$ '«humidité» ont un sens géomorphologique plus précis que dans celui de MicheL.

${ }^{5}$ E Etant donné qu'elles sont représentées par des formes de relief (paysages à inselberg), des phases morphogénétiques «humides» (phases d'activité humide), d'une morphogenèse différente de celle qui caractérise les régions arides, doivent avoir existé. (《Alteritisches Tieferschalten», RUST, 1970). Notre terminologie est limitée aux phases d'activité et de stabilité des régions arides. 


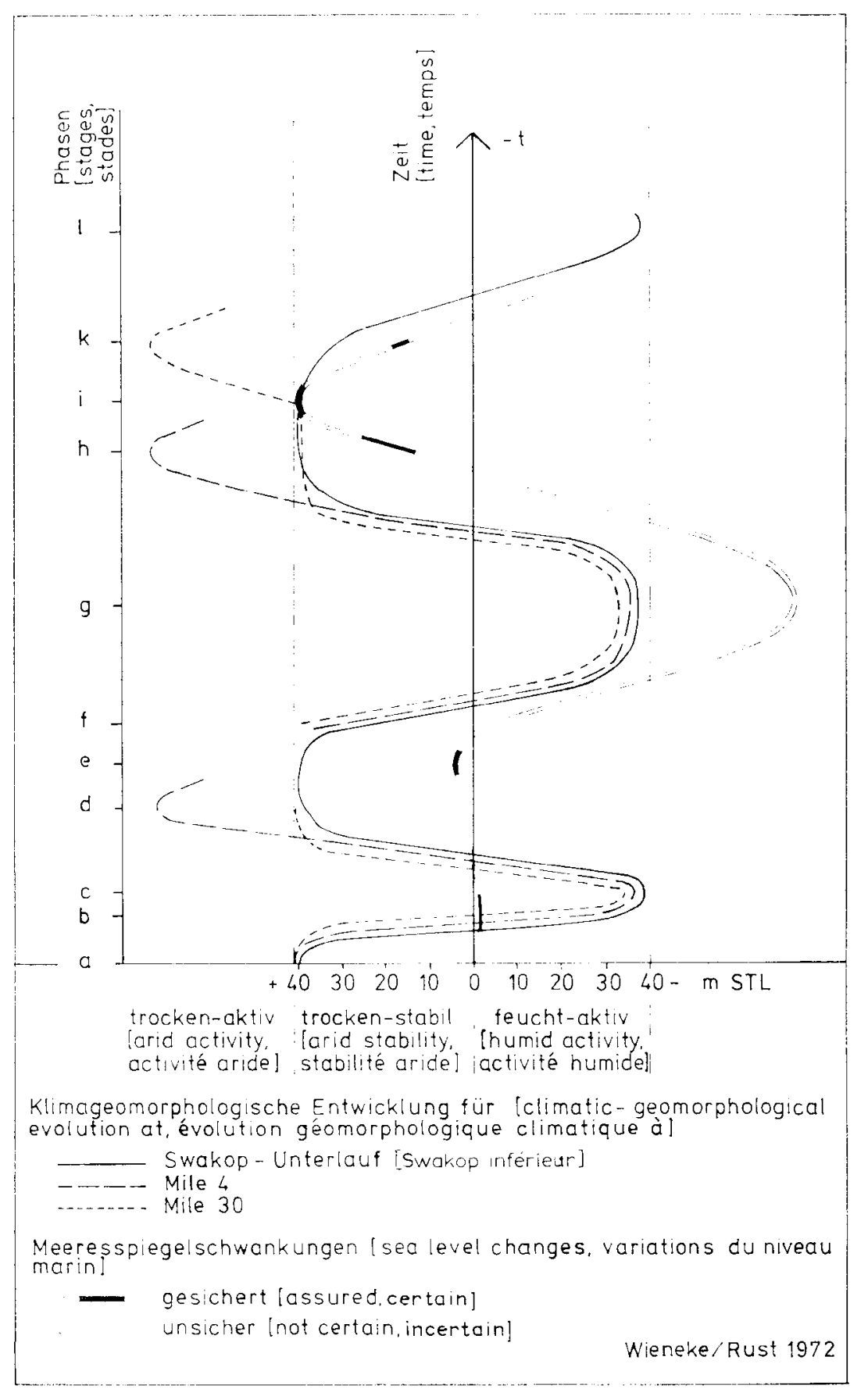

Fig. 6--- Variations du niveau marin et évolution morphoclimatique dans le désert du Namib central.
63

Le diagramme montre que l'évolution des formes du relief du Namib Central se fonde bien sur une alternance de phases d'activité humide et de stabilité aride, c'est-à-dire sur une alternance de périodes d'activité fluviatile à la fois autochtone et allogène et d'activité fluviatile allogène combinée avec le type de pédogenèse décrit dans le tableau Ir. Il y eut aussi de rares phases d'activité aride où les processus éoliens dominaient. Plus au Sud, à la limite septentrionale actuelle du Namib dunaire, on pourrait reconnaître davantage de phases d'activité aride dans la morphogenèse quaternaire.

A Mile 4, au Nord du Swakop, nous avons trouvé deux phases d'activité aride ( $h$ et d), ce qui signifie que le Swakop inférieur a été au moins deur fois «tsondabisé», c'est-à-dire que son cours inférieur a été barré par des dunes comme c'est lc cas actuel de la rivière Tsondab. Plus au Nord, à Mile 30, au lieu de phases d'activité aride, nous n'avons pu prouver que des phases de stabilité aride. Ainsi cette conception nouvelle permet de distinguer des variétés régionales de régions arides en se fondant sur des critères morphogénétiques: on a pu fixer la limite septentrionale de l'activité aride qui passe entre les localités Mile 4 ct Mile 30 .

\section{FRIEDRICH WIENEKE ET UWE RUST}

\section{SUMMARY}

We studied the Quaternary land form evolution of Central Namib Desert as the result of a complex interaction of marine-litoral, fluvial and eolian geomorphic processes.

We found two high stands of the sea level (at $17 \mathrm{~m}$ and at $2 \mathrm{~m}$ above storm tide level) and one subrecent low stand. We could prove several «climatical» changes for the region studied by pedological, sedimentological and geomorphological methods, and were are able to relate them by their ages to the fossil sea levels. High sea levcls coincide with «arid» climatic stages, low sea levels with «humid» stages.

From the point of view of a gemorphologist we had to criticize that certain land form types used to be directly connected with the climatological terms «arid» and «humid». This leads to contradictions in a «climatic» interpretation of land forms. Therefore we had to develop a special terminology and a new concept for the «climatic geomorphic stages» in the arid region we studied. We defined the 
following climatic geomorphic stages in order to describe ecologically a more profound relation between geomorphic processes and their «climatic» conditions: arid activity, arid stability, and humid activity The quaternary evolution of land forms of Central Namib Deser consists essentially of a repeated alternation of the phases of arid stability and of humid activity. In the south, at the northern limit of Dune Namib, phases of arid activity have been of a greater importance.

\section{BIBLIOGRAPHIE}

BAULIG, H. (1956) - Vocabulaire franco-anglo-allemand de géomorphologie, Publ. Fac. Lettres Univ. Strasbourg, fasc. 130, Paris, $230 \mathrm{p}$. DAvies, O. (1959) - «Pleistocene raised beaches in South-West Africa», Congr. Geol. Intern. XX, Mexico 1956, Asociación de Servicios Geológicos Africanos, Actas y Trabajos de las Reuniones Celebradas en Mexico en 1956, México, pp. 347-350.

ELouard, P. (1967) - «Eléments pour une définition des principaux niveaux du Quaternaire sénégalo-mauritanien. I. Plage à Ar'ca senilis», Bull. I. F. A. N., t.xxIx, sér. A, n." 2, Dakar, pp. 822-836.

HAGEDORN, H. (1971) - Untersuchungen über Relieftypen arider Räume an Beispielen aus dem Tibesti-Gebirge und seiner Umgebung. Zeitschr. f. Geomorphologie, NF Suppl.-Bd. 11, Berlin, 220 p.

JAEGER, F. (1965) - Geographische Landschaften Südwestafrikas. Wiss. Forschungen in Südwestafrika, 2. Folge, Windhoek, $251 \mathrm{p}$.

Michel, P. (1967) --.- «Les dépôts du Quaternaire récent dans la basse vallée du Sénégal», Bull. I. F. A. N., t. xxIx, sér. A, n." 2, Dakar, pp. $853-860$.

MicheL, P. (1968) - «Genèse et évolution de la vallée du Sénégal, de Bakel à l'embouchure, Afrique Occidentale», Zeitschr. f. Geomorphologie, NF Bd. 12, pp. 318-349.

RoHdenburG, H. (1970)---«Morphodynamische Aktivitäts- und Stabilitätszeiten statt Pluvial- und Interpluvialzeiten», Eiszeitalter $u$. Gegenwart, Bd, 21, pp. 81-96.

Russel, R. J. (1962) - «Origin of beach rock», Zeitschr. f. Geomorphologie, NF Bd. 6, pp. 1-17.

Rust, U. (1970) - Beiträge zum Problem der Inselberglandschaften aus dem Mittleren Südwestafrika. Hamburger Geogr. Studien, H. 23 , Hamburg, $280 \mathrm{p}$.

Rust, U. et WiEnekE, F. (1973a) - «Grundzüge der quartären Reliefentwicklung der Zentralen Namib, Südwestafrika (Erste ausgewählte Ergebnisse einer Forschungsreise 1972)», Journal SWA Wiss. Ges., Bd. XXVII (sous presse).

Rust, U. et WIENEKE, F. (1973b)-- «Die Rundungsgradanalyse nach Reichelt als Feldmethode in Trockengebieten», Petermanns Geogr. Mitt. (sous presse).

Scholz, H. (1963) - Studien über die Bodenbildung zweischen Rehoboth und Walvis Bay. Diss. Landwirtschaftliche Fakultät Bonn, 184 p.
Soares De Carvalho, G. (1961) - Geologia do Deserto de Moçâmedes (Angola). Mem. Junta Invest. Ultramar, sér. A, n." 26, 227 p.

SPREITZER, H. (1965) - Beobachtungen zur Geomorphologie der Zentralen Namib und ihrer Randgebiete, Sonderveröffentlichung SWA Wiss. Ges. Windhoek, n." 4, 34 p.

TricarT, J. (1961) - Notice explicative de la Carte Géomorphologique du delta du Sénégal. Mémoires du BRGM, n. ${ }^{\circ} 8,137 \mathrm{p}$.

TricarT, J. et CAIlleuX, A. (1965) - Traité de géomorphologie. T. I. Introduction à la géomorphologie climattique. Paris, $306 \mathrm{p}$.

WEISCHET, W. (1970) - «Diskussionsbemerkung zum Vortrag Mensching Tagungsbericht u. Wiss. Abh.», Deutscher Geographentag Kiel, 1969 , p. 570 .

WIENEKE, F. et RUST, U. (1972) - «Das Satellitenbild als Hilfsmittel zur Formulierung geomorphologischer Arbeitshypothesen (Beispiel Zentrale Namib, Südwestafrika)», Wiss. Forschungen in Südwestafrika, 11. Folge, Windhoek, $16 \mathrm{p}$.

WIEneke, F. et RUST, U. (1973) - - KKlimageomorphologische Phasen in der Zentralen Namib (Südwestafrika»), Mitt. Geogr. Ges. München, Bd. 58 (sous presse). 